\title{
Ginsenoside Rb1 attenuates intestinal ischemia/reperfusion-induced inflammation and oxidative stress via activation of the PI3K/Akt/Nrf2 signaling pathway
}

\author{
SUFANG CHEN $^{1 *}$, XIANG LI $^{1 *}$, YANLING WANG ${ }^{1}$, PANWEI MU ${ }^{2}$, CHAOJIN CHEN ${ }^{1}$, \\ PINJIE HUANG ${ }^{1}$ and DEZHAO LIU ${ }^{1,2}$ \\ Departments of ${ }^{1}$ Anesthesiology and ${ }^{2}$ Endocrinology, The Third Affiliated Hospital of \\ Sun Yat-sen University, Guangzhou, Guangdong 510630, P.R. China
}

Received June 16, 2018; Accepted February 12, 2019

DOI: $10.3892 / \mathrm{mmr} .2019 .10018$

\begin{abstract}
Ginsenoside Rb1 (GRb1), one of the major active saponins isolated from ginseng, has recently been reported to protect various organs against ischemia/reperfusion (IR) injury; however, the mechanisms underlying these protective effects following intestinal IR (IIR) remain unclear. The present study aimed to evaluate the effects of GRb1 on IIR injury and determine the mechanisms involved in these effects. Sprague Dawley rats were subjected to $75 \mathrm{~min}$ of superior mesenteric artery occlusion, followed by $3 \mathrm{~h}$ of reperfusion. GRb1 (15 mg/kg) was administered intraperitoneally $1 \mathrm{~h}$ prior to the induction of IIR, with or without intravenous administration of Wortmannin [WM; a phosphoinositide 3-kinase (PI3K) inhibitor, $0.6 \mathrm{mg} / \mathrm{kg}$ ]. The degree of intestinal injury and oxidative stress-induced damage was determined by histopathologic evaluation and measurement of the serum activity levels of D-lactate, diamine oxidase and endotoxin, and the levels of malondialdehyde (MDA), superoxide dismutase (SOD) and 8-iso-prostaglandin $\mathrm{F}_{2 \alpha}$ (8-iso- $\mathrm{PGF}_{2 \alpha}$ ).
\end{abstract}

Correspondence to: Professor Dezhao Liu, Department of Anesthesiology, The Third Affiliated Hospital of Sun Yat-sen University, 600 Tianhe Road, Guangzhou, Guangdong 510630, P.R. China

E-mail: sumsldz@126.com

Dr Panwei Mu, Department of Endocrinology, The Third Affiliated Hospital of Sun Yat-sen University, 600 Tianhe Road, Guangzhou, Guangdong 510630, P.R. China

E-mail: mpwsums@126.com

"Contributed equally

Abbreviations: GRb1, ginsenoside Rb1; IIR, intestinal ischemia/reperfusion; SMA, superior mesenteric artery; PI3K, phosphoinositide 3-kinase; Akt, protein kinase B; WM, Wortmannin; DAO, diamine oxidase; MDA, malondialdehyde; SOD, superoxide dismutase; Nrf2, nuclear factor erythroid 2-related factor 2; 8-iso- $\mathrm{PGF}_{2 \alpha}, 8$-iso-prostaglandin $\mathrm{F}_{2 \alpha}$

Key words: GRb1, PI3K, IIR injury, Nrf2
The protein expression levels of p85, phosphorylated (p)-p85, protein kinase B (Akt), p-Akt and nuclear factor erythroid 2-related factor 2 (Nrf2) were determined via western blotting, and the concentrations of tumor necrosis factor- $\alpha$ (TNF- $\alpha$ ), interleukin (IL)-1 $\beta$ and IL-6 were measured via ELISA. It was revealed that IIR led to severe intestinal injury (as determined by significant increases in intestinal Chiu scores), which was accompanied with disruptions in the integrity of the intestinal mucosal barrier. IIR also increased the expression levels of TNF- $\alpha$, IL-1 $\beta$, IL-6, MDA and 8-iso-PGF $2 \alpha$ in the intestine, and decreased those of SOD. GRb1 reduced intestinal histological injury, and suppressed inflammatory responses and oxidative stress. Additionally, the protective effects of GRb1 were eliminated by WM. These findings indicated that GRb1 may ameliorate IIR injury by activating the PI3K/protein kinase B/Nrf2 pathway.

\section{Introduction}

Small intestinal ischemia/reperfusion (IIR) injury is a common and serious pathological condition that occurs during numerous clinical events, including superior mesenteric artery (SMA) occlusion, liver transplantation and hemorrhagic shock (1). Furthermore, IIR injury may lead to severe damage to remote organs, including the lungs, kidneys and liver, thereby adversely affecting the prognosis of patients $(2,3)$. Improved treatments have been increasingly used in clinical settings; however, the morbidity and mortality rates of IIR injury remain high (4). Consequently, it is urgent to understand the mechanisms underlying IIR injury and identify novel therapeutic approaches in the treatment of this condition.

Ginsenoside Rb1 (GRb1) is a major active ingredient of Panax ginseng C.A. Meyer (Araliaceae family), a traditional herbal medicine that is widely used in Asian countries (5). GRb1 has been reported to protect various organs from IIR injury due to its antioxidant and antiapoptotic effects $(6,7)$. GRb1 increases phosphorylated (p)-protein kinase B (Akt) levels and promotes $\mathrm{p}$-extracellular signal-regulated kinase $1 / 2$-mediated signaling to suppress amyloid $\beta(\mathrm{A} \beta)$-induced apoptosis (5), whereas exposure to $A \beta$ leads to the accumulation of reactive oxygen species (ROS) and lipid 
peroxidation. Furthermore, GRb1 protected neurons against high glucose-induced neurotoxicity by inhibiting oxidative stress and mitochondrial dysfunction (8); however, it has not yet been determined whether GRb1 can attenuate IIR injury, and the underlying mechanisms remain unknown.

Phosphoinositide 3-kinase (PI3K), a member of the phospholipid kinase family, serves important roles in the regulation of the apoptosis, proliferation, differentiation and metabolism of cells (9). The serine-threonine protein kinase Akt is a downstream target of PI3K; when stimulated by extracellular signals, PI3K-activated Akt initiates a cascade of intracellular reactions (10). PI3K is composed of a regulatory subunit (p85) and a catalytic subunit (p110), and activation of the catalytic subunit depends upon the phosphorylation of p85 (11). Activation of p85 by phosphorylation leads to the phosphorylation of Akt (12). Nuclear factor erythroid 2-related factor 2 (Nrf2) is an important regulator of the expression of antioxidant enzymes and enhancement of endogenous antioxidant capacity (13). Previous studies have reported that the nuclear translocation of Nrf2 requires the activation of the PI3K/Akt pathway $(14,15)$; however, the effects of GRb1 and the associated PI3K/Akt pathway on IIR injury require further investigation.

In the present study, an SMA occlusion/reperfusion model was generated in rats to induce IIR injury and Wortmannin (WM) was used to inhibit the PI3K/Akt signaling pathway. Rats were subsequently treated with GRb1 to investigate whether GRb1 attenuates IIR injury by activating the PI3K/Akt/Nrf2 pathway.

\section{Materials and methods}

Animals. The experimental protocol and design were approved by the Institutional Animal Care and Use Committee of Sun Yat-sen University (Guangzhou, China), and were conducted in accordance with the Chinese guidelines for humane treatment of animals (16). A total of 30 male Sprague Dawley rats (aged 8 weeks, 200-250 g), were purchased from the Animal Center of Guangdong Province (Guangzhou, China). The rats were housed individually in cages under pathogen-free conditions for 1 week prior to surgery, and maintained under controlled temperature $\left(20-23^{\circ} \mathrm{C}\right)$, humidity (45-55\%) and light (12:12-h light/dark cycle) conditions with access to food and water ad libitum. The rats were randomized into five groups ( $n=6$ per group): A sham-operated group (Sham); an ischemia/reperfusion (IR) of the intestine group; a GRb1 + IIR group (IR + GRb1); a WM + IIR group (IR + WM) and a GRb1 $+\mathrm{WM}+\mathrm{IIR}$ group (IR + GRb1 + WM). All rats were anaesthetized via intraperitoneal injection of $10 \%$ chloral hydrate (350 mg/kg) (2) following fasting for $16 \mathrm{~h}$ prior to the surgical procedure, and the abdomen was opened with a midline incision with animals in the supine position. In the IR group, the abdomen was opened, and the SMA was isolated and clamped for $75 \mathrm{~min}$; then, the clamp was released to maintain the rats for $3 \mathrm{~h}$ during reperfusion (17). For sham treatment, the SMA was isolated but not clamped, and was maintained in this state for the same period during the surgical procedure. In the other three groups, the rats subjected to IIR were intraperitoneally injected with GRb1 (15 mg/kg; Shanghai Tauto Biotech Co., Ltd., Shanghai, China) (18) and/or intravenously injected with
WM (0.6 mg/kg; Sigma-Aldrich; Merck KGaA, Darmstadt, Germany) (19) $1 \mathrm{~h}$ prior to the operation. Conversely, rats of the Sham and IR groups received the same volume physiological saline. During the operation, the body temperature of all rats was maintained at $38^{\circ} \mathrm{C}$ using heated pads, and $10 \mathrm{ml} / \mathrm{kg}$ physiological saline $\left(37^{\circ} \mathrm{C}\right)$ was injected subcutaneously to minimize the risk of dehydration following closure of the abdomen.

Small intestinal mucosa collection. Following completion of the experiment, the rats were anesthetized via intraperitoneal injection of $10 \%$ chloral hydrate $(400 \mathrm{mg} / \mathrm{kg}$ ) (2) and sacrificed by decapitation. A segment $(1.0 \mathrm{~cm})$ of intestine was extracted at a point $10 \mathrm{~cm}$ from the terminal ileum, fixed in $10 \%$ formaldehyde for $24 \mathrm{~h}$ at $4^{\circ} \mathrm{C}$, and then embedded in paraffin for sectioning. The remainder of the small intestine was stored at $-80^{\circ} \mathrm{C}$ until further analysis.

Intestinal histological examination. Slices (5- $\mu \mathrm{m}$ thickness) were prepared from the paraffin-embedded intestinal tissue and stained with hematoxylin and eosin (H\&E) at $37^{\circ} \mathrm{C}$ for 3 and $5 \mathrm{~min}$, respectively. The extent of damage to the intestinal mucosa was subsequently characterized by two histologists (who were initially blinded to the experiment), according to Chiu's classification (20). The Chiu grading system criteria comprise 5 subdivisions, according to alterations to the villi and glands of intestinal mucosa: Grade 0, normal mucosa; grade 1, development of subepithelial Gruenhagen's space at the tip of the villi; grade 2, extension of the space with moderate epithelial lifting; grade 3 , massive epithelial lifting with a number of denuded villi; grade 4, denuded villi with exposed capillaries and grade 5, disintegration of the lamina propria, ulceration and hemorrhage.

Measurement of D-lactate, diamine oxidase (DAO) and endotoxin levels in the serum. Portal vein blood samples were collected at the end of the experiment, and the serum was obtained via centrifugation at $3,000 \mathrm{x}$ for $15 \mathrm{~min}$ at $4^{\circ} \mathrm{C}$ and then stored at $-80^{\circ} \mathrm{C}$ prior to analysis of D-lactate, DAO and endotoxin levels. The serum levels of D-lactate (AF7304-SP) and DAO (8298-AO-010) were determined using an enzymatic spectrophotometric assay with reagent kits according to the manufacturer's protocols (Sigma-Aldrich; Merck KGaA). The serum levels of endotoxin (YX1214) were determined using a Limulus Amebocyte Lysate Assay kit (Shanghai Biochemical Co., Ltd., Shanghai, China) according to the manufacturer's protocols.

ELISA. The intestinal tissue homogenates were centrifuged at $4,000 \mathrm{x} g$ for $15 \mathrm{~min}$ at $4^{\circ} \mathrm{C}$, and the supernatants were transferred into fresh tubes for further analysis. Briefly, the total amount of intestinal protein was quantified using a Bicinchoninic Acid Protein Assay kit (Guangzhou Scissorhands Gene Technology Co., Ltd., Guangzhou, China). The concentrations of tumor necrosis factor- $\alpha$ [TNF- $\alpha$; MM-0180R2, interleukin (IL)-6 (MM-0163M1), IL-1 $\beta$ (MM-0040M1)] and 8-iso-prostaglandin $\mathrm{F}_{2 \mathrm{a}}$ (8-iso-PGF $\mathrm{Pa}_{2 \mathrm{a}}$ MM-43647M1) were measured using corresponding commercial ELISA kits (Guangzhou Scissorhands Gene Technology Co., Ltd.) according to the manufacturer's protocols. Absorbance at $450 \mathrm{~nm}$ was measured using an 
EL340 Biokinetics microplate reader (BioTek Instruments, Inc., Winooski, VT, USA). The levels of 8-iso-PGF $2 \alpha$, TNF- $\alpha$, IL-1 $\beta$ and IL-6 were calculated in pg/mg.

Quantification of malondialdehyde (MDA) and superoxide dismutase (SOD) activity in the small intestinal mucosa. Samples of small intestinal mucosa was homogenized with normal saline, frozen at $-20^{\circ} \mathrm{C}$ for $5 \mathrm{~min}$, and centrifuged at $4,000 \mathrm{x} \mathrm{g}$ for $15 \mathrm{~min}$ at $4^{\circ} \mathrm{C}$. The supernatants were transferred into fresh tubes for evaluation of the MDA levels and SOD activity at $37^{\circ} \mathrm{C}$ with thiobarbituric acid and SOD detection kits (Nanjing Jiancheng Bioengineering Institute, Nanjing, China), respectively, according to the manufacturer's protocols. The final concentration of MDA in the intestinal mucosa was calculated in $\mathrm{nmol} /(\mathrm{mg}$ protein), and the levels of SOD activity were calculated in $\mathrm{U} /(\mathrm{mg}$ protein).

Western blotting analysis. Total protein was extracted from the intestinal mucosa using ice-cold radio immunoprecipitation assay buffer (Sigma-Aldrich, Merck KGaA) and the protein concentration was quantified using a Bicinchoninic Acid Protein Assay kit (Sigma-Aldrich, Merck KGaA). Then, protein samples $(50 \mu \mathrm{g} /$ lane $)$ were separated via $10 \%$ SDS-PAGE and transferred onto polyvinylidene fluoride membranes (EMD Millipore, Billerica, MA, USA). Membranes were blocked with 5\% bovine serum albumin (Cell Signaling Technology, Inc., Danvers, MA, USA) for $1 \mathrm{~h}$ at $37^{\circ} \mathrm{C}$. The membranes were subsequently incubated with the following primary antibodies overnight at $4^{\circ} \mathrm{C}$ (all from Santa Cruz Biotechnology, Inc., Dallas, TX, USA): Anti-p85 (sc-1637; 1:1,000); anti-p-p85 (1:1,000; sc-12929); anti-Akt (1:1,000; sc-5298); anti-p-Akt (1:1,000; sc-293125); anti-Nrf2 $(1: 1,000 ;$ sc-722) and anti-GAPDH $(1: 1,000 ;$ sc-47724). The membranes were subsequently washed with $5 \%$ non-fat milk in TBS containing 1\% Tween-20, and incubated with horseradish peroxidase-conjugated secondary antibody $(1: 2,000$; HAF019, Santa Cruz Biotechnology, Inc.) for $1 \mathrm{~h}$ at $37^{\circ} \mathrm{C}$. GAPDH was used as an internal control. Protein bands were visualized using an enhanced chemiluminescence detection system (Nanjing KeyGen Biotech Co., Ltd., Nanjing, China) and protein expression was quantified using ImageJ software 2.x (National Institutes of Health, Bethesda, MD, USA).

Statistical analysis. All experiments were repeated in triplicate and all data were expressed as the mean \pm standard deviation. Analysis was performed using GraphPad Prism version 5.0 (GraphPad Software, Inc., La Jolla, CA, USA). One-way analyses of variance were performed for multiple comparisons, followed by post-hoc Bonferroni tests to compare unpaired values. $\mathrm{P}<0.05$ was considered to indicate a statistically significant difference.

\section{Results}

GRbl attenuates IIR-induced pathological alterations in the intestine. It has previously been reported that GRb1 ameliorates lung injury and cardiac IR injury due to its antioxidative properties $(21,22)$. In the present study, it was demonstrated that $75 \mathrm{~min}$ of ischemia, followed by $3 \mathrm{~h}$ of reperfusion, induced severe damage to the small intestinal mucosa. The villi and glands appeared normal in the Sham group, with no neutrophil infiltration detected in the mucosal epithelial layer, whereas numerous erosion and bleeding sites were observed in the IR group (Fig. 1A and B). It was revealed that pre-treatment with GRb1 significantly attenuated the extent of small intestinal injury; only mild edema of the mucosal villi and a small number of necrotic epithelial cells were observed in the mucosal epithelial layer (Fig. 1C). Conversely, WM exacerbated IIR injury, with increased erosion and bleeding, and neutrophil infiltration was promoted in the intestinal mucosa (Fig. 1D). Furthermore, the protective effects of GRb1 against IIR injury were markedly attenuated by WM (Fig. 1E). In accordance with the alterations in intestinal morphology, the Chiu scores were significantly increased in the IR group compared with the Sham group $(\mathrm{P}<0.05$; Fig. $1 \mathrm{~F})$. Treatment with GRb1 prior to ischemia significantly reduced the Chiu score $(\mathrm{P}<0.05)$, whereas WM treatment significantly increased the Chiu score compared with the IR group $(\mathrm{P}<0.05)$. The results suggested that pre-treatment with GRb1 attenuated IIR-induced pathological alterations in the small intestine and that WM, an inhibitor of the PI3K/Akt signaling pathway, suppressed the protective effects of GRb1.

GRb1 increases the integrity of the intestinal mucosal barrier following IIR injury. The serum levels of D-lactate, DAO and endotoxin were determined in blood samples obtained from the portal vein. High serum levels of D-lactate and DAO indicate increased permeability of the intestinal mucosa (23), whereas the levels of endotoxin are associated with the degree of injury to the intestinal mucosal barrier (24). As presented in Fig. 2, it was demonstrated that the serum levels of D-lactate, DAO and endotoxin were significantly increased in the IR group compared with the Sham group, indicating that IIR disrupted the integrity of the intestinal mucosal barrier $(\mathrm{P}<0.05)$. Pre-treatment with GRb1 significantly decreased the serum levels of D-lactate, DAO and endotoxin compared with the IR group $(\mathrm{P}<0.05)$, whereas pre-treatment with WM further increased the levels of these factors compared with the IR group $(\mathrm{P}<0.05)$. There were no significant differences in the serum levels of D-lactate, DAO or endotoxin between the IR and $\mathrm{IR}+\mathrm{GRb} 1+\mathrm{WM}$ groups $(\mathrm{P}>0.05)$. The results suggested that the effects of IIR on intestinal injury and mucosal permeability were attenuated by GRb1, whereas inhibition of the PI3K/Akt signaling pathway promoted further damage to the intestinal mucosa.

GRbl suppresses proinflammatory cytokine secretion following IIR injury. IIR injury is characterized by the secretion of proinflammatory cytokines and the infiltration of neutrophils into the intestinal mucosa (25). As presented in Fig. 3, the expression levels of intestinal TNF- $\alpha$, IL-6 and IL-1 $\beta$ in rats subjected to IIR were significantly increased compared with the Sham group $(\mathrm{P}<0.05)$, consistent with our previous findings (26). Furthermore, pre-treatment with WM promoted further increases in the expression levels of TNF- $\alpha$, IL- 6 and IL-1 $\beta$ compared with the IR group $(\mathrm{P}<0.05)$; conversely, GRb1 significantly downregulated the expression of the aforementioned proinflammatory cytokines following IIR $(\mathrm{P}<0.05)$. The results indicated that administration of $15 \mathrm{mg} / \mathrm{kg}$ GRb1 attenuates the secretion of proinflammatory 
A

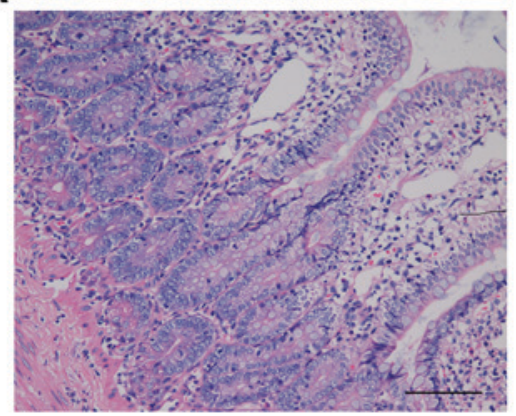

D
B

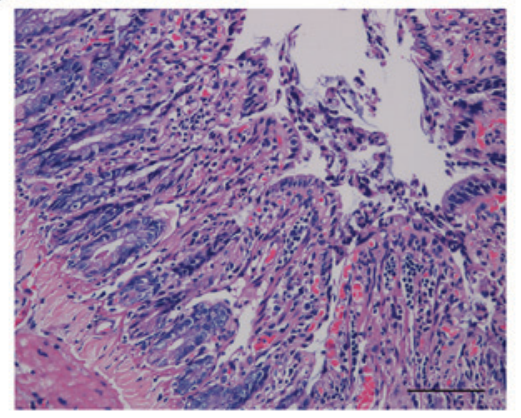

C

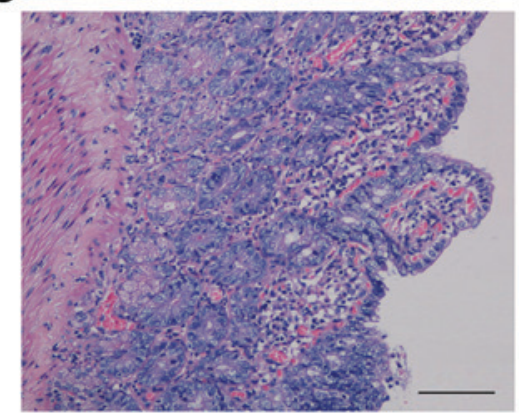

E
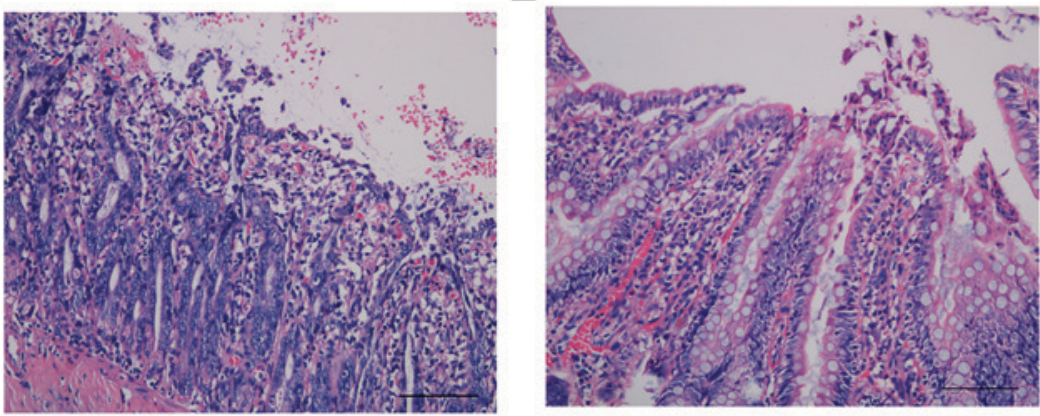

F

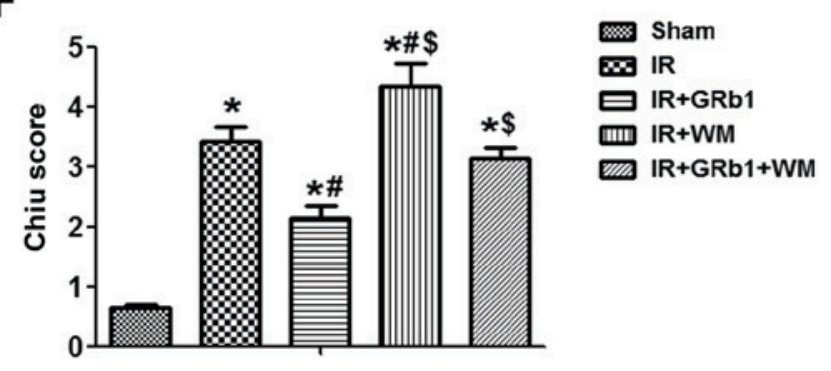

Figure 1. Morphological analysis of intestinal sections and histological scoring. Representative images of H\&E staining (magnification, $\mathrm{x} 200)$ of the intestines of rats from the (A) Sham, (B) IR (75 min intestinal ischemia followed by $3 \mathrm{~h}$ reperfusion), (C) IR + GRb1 (15 mg/kg), (D) IR + WM (0.6 mg/kg) and (E) IR + GRb1 + WM groups. (F) Chiu score for the quantification of intestinal damage following IR. N=6/group. Data are presented as the mean \pm standard deviation. " $\mathrm{P}<0.05$ vs. Sham, " $\mathrm{P}<0.05$ vs. IR, ${ }^{\$} \mathrm{P}<0.05$ vs. IR + GRb1. GRb1, ginsenoside Rb1; IR, ischemia/reperfusion; Sham, sham-operated group; WM, Wortmannin.

cytokines and the activation of neutrophils, whereas WM had opposing effects.

GRb1 attenuates oxidative stress induced by IIR injury. It has previously been reported that IIR mediates acute lung injury via increases in free radical species production and oxidative stress-induced lipid peroxidation (27). In the present study, the mechanisms underlying the protective effects of the PI3K/Akt signaling pathway on IIR injury were investigated. Consistent with the aforementioned findings, $75 \mathrm{~min}$ of ischemia followed by $3 \mathrm{~h}$ of reperfusion significantly increased the intestinal levels of MDA and 8-iso- $\mathrm{PGF}_{2 \alpha}$, but reduced the activity levels of SOD compared with the Sham group ( $\mathrm{P}<0.05$; Fig. 4). Additionally, WM significantly increased the levels of MDA and 8-iso-PGF ${ }_{2 \alpha}$, and decreased the activity of SOD compared with the IR group $(\mathrm{P}<0.05)$, whereas GRb1 had opposing effects $(\mathrm{P}<0.05)$. Collectively, the results suggested that GRb1 alleviates intestinal injury during IIR via the inhibition of oxidative stress.
Pre-treatment with GRbl activates the PI3K/Akt/Nrf2 signaling pathway in IIR injury. It has previously been reported that the PI3K/Akt signaling pathway is associated with injury following IIR $(28,29)$. In the present study, the association between PI3K/Akt signaling and the protective effects of GRb1 were investigated by determining the intestinal expression levels of $\mathrm{p} 85$, Akt and Nrf2 following IIR. As presented in Fig. 5, IIR induced the activation of PI3K, as determined by the significantly increased phosphorylation of $\mathrm{p} 85$ compared with the Sham group $(\mathrm{P}<0.05)$. Similarly, the relative expression of intestinal p-Akt in the IR group was significantly increased compared with in the Sham group $(\mathrm{P}<0.05)$. Pre-treatment with GRb1 further increased the levels of p-p85 and p-Akt compared with the IR group, whereas WM had opposing effects. Additionally, the expression levels of Nrf2 were significantly increased following pre-treatment with GRb1 compared with the IR group $(\mathrm{P}<0.05)$. Conversely, pre-treatment with WM significantly decreased the levels of Nrf2 expression compared with the IR group $(\mathrm{P}<0.05)$. No 
A
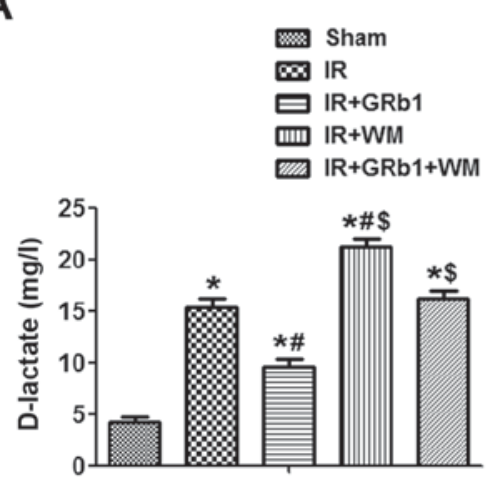

B
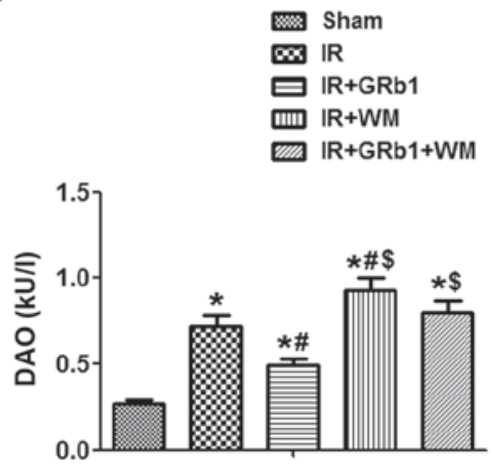

C
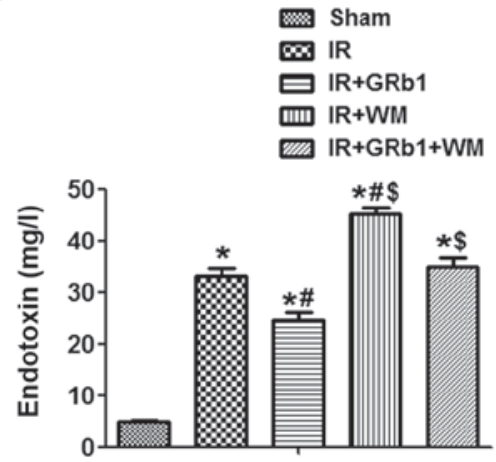

Figure 2. Integrity of the intestinal mucosal barrier following IIR injury. Serum levels of (A) D-lactate, (B) DAO and (C) endotoxin following Sham or IR treatment in the presence or absence of $15 \mathrm{mg} / \mathrm{kg} \mathrm{GRb1}$ and $0.6 \mathrm{mg} / \mathrm{kg}$ WM. IIR decreased the integrity of the intestinal mucosal barrier, as demonstrated by significant increases in the levels of serum D-lactate, DAO and endotoxin. N=6/group. Data are presented as the mean \pm standard deviation. * $\mathrm{P}<0.05$ vs. Sham, ${ }^{\#} \mathrm{P}<0.05$ vs. IR, ${ }^{\$} \mathrm{P}<0.05$ vs. IR + GRb1. DAO, diamine oxidase; GRb1, ginsenoside Rb1; IIR, intestinal ischemia/reperfusion; IR, ischemia/reperfusion; Sham, sham-operated group; WM, Wortmannin.

A

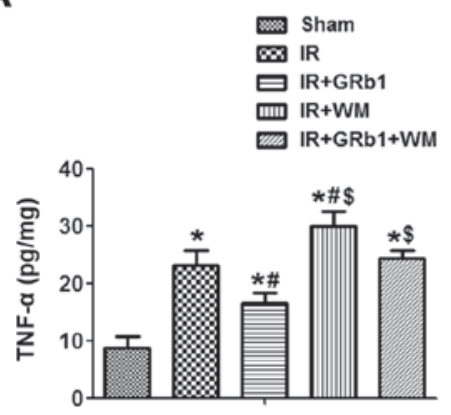

B

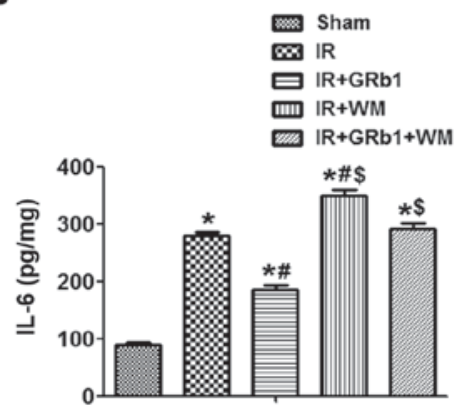

C

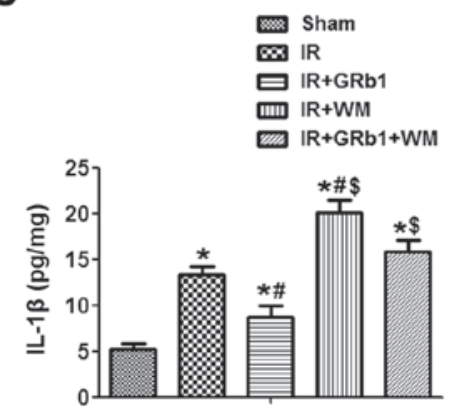

Figure 3. GRb1 attenuates inflammatory responses in intestinal mucosa following intestinal IR injury. Intestinal expression levels of (A) TNF- $\alpha$, (B) IL-6 and (C) IL-1 $\beta$ following Sham or IR treatment, in the presence or absence of $15 \mathrm{mg} / \mathrm{kg}$ GRb1 and $0.6 \mathrm{mg} / \mathrm{kg}$ WM. Data are presented as the mean \pm standard deviation. ${ }^{*} \mathrm{P}<0.05$ vs. Sham, ${ }^{\sharp} \mathrm{P}<0.05$ vs. IR, ${ }^{\$} \mathrm{P}<0.05$ vs. IR + GRb1. GRb1, ginsenoside Rb1; IL-1 $\beta$, interleukin-1 $\beta$; IL-6, interleukin-6; IR, ischemia/reperfusion; Sham, sham-operated group; TNF- $\alpha$, tumor necrosis factor- $\alpha$; WM, Wortmannin.

significant differences in the levels of phosphorylation or Nrf2 expression were observed between the IR and IR + GRb1 + WM groups $(\mathrm{P}>0.05)$. Collectively, the results suggests that the PI3K/Akt/Nrf2 signaling pathway is involved in mediating the protective effects of GRb1 against injury following IIR.

\section{Discussion}

IIR injury is a leading cause of morbidity and mortality in various diseases, as it may induce serious damage in nearby and remote organs, and lead to multiorgan dysfunction (30). Therefore, an improved understanding of the pathophysiological mechanisms underlying IIR injury may lead to more effective prophylaxis and treatment of this condition.

A previous study reported that the 7-day survival of Sprague Dawley rats treated with 75 min of SMA occlusion followed by $3 \mathrm{~h}$ of reperfusion was $41.7 \%$ (2). In the present study, it was demonstrated that this treatment induced substantial IIR injury in a rat model, as determined by the pathological morphology observed in the intestine and increased Chiu scores. These findings were consistent with a previous study (31), and indicated that the rat model of IIR injury was well-established.

Ginseng has been one of the most extensively used herbal medicines in eastern Asian countries for $>2,000$ years (32).
Ginsenoside is the major pharmacologically active ingredient of ginseng and has been noted for its biological properties, including antioxidation, signal transduction and interactions with receptors (33). GRb1, one of the principal bioactive ingredients in ginsenoside, exhibits pharmacological properties, including anti-inflammation, antifatigue and neuroprotection (34-36). Various studies have reported that GRb1 protects against the IR-induced injury of numerous organs, including the heart and kidneys $(37,38)$. Additionally, activation of the PI3K/Akt pathway attenuates injury and alleviates damage to organs, such as the intestine and lungs $(39,40)$. The effects of GRb1 on IIR injury and the underlying mechanisms have not yet been fully investigated. In the present study, it was demonstrated that GRb1 attenuates IIR injury in rats, potentially by suppressing inflammatory responses and oxidative stress. The effects of GRb1 on the PI3K/Akt pathway were determined via western blotting. It was revealed that the relative expression of p-p85 in the IR + GRb1 group was increased compared with in the IR group. Activation of p85 promotes the phosphorylation of Akt; the levels of p-Akt expression were also significantly upregulated in the IR + GRb1 group compared with in the IR group. Collectively, these findings indicated that GRb1 attenuates IIR-induced inflammation and oxidative stress by activating the PI3K/Akt pathway. 
A
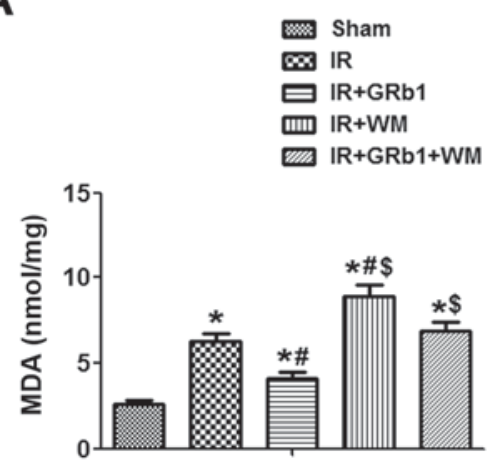

B
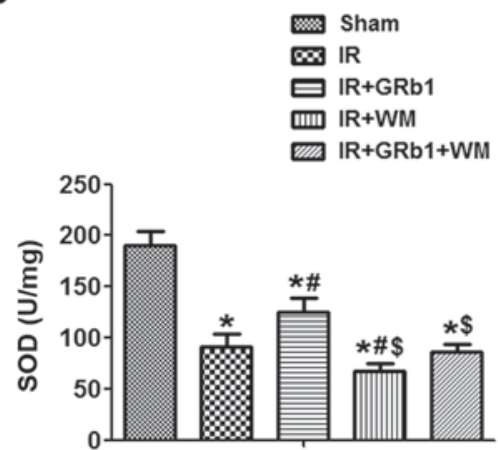

C

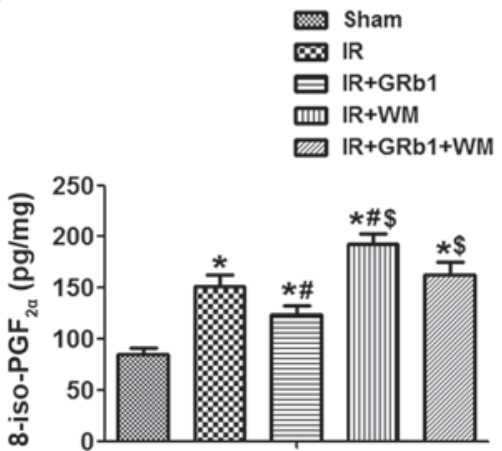

Figure 4. GRb1 reduces oxidative stress in intestinal mucosa following intestinal IR injury. Intestinal levels of (A) MDA content, (B) SOD activity and (C) 8-iso-PGF $2 \alpha$ expression following Sham or IR treatment, in the presence or absence of $15 \mathrm{mg} / \mathrm{kg} \mathrm{GRb1} \mathrm{and} 0.6 \mathrm{mg} / \mathrm{kg}$ WM. N=6/group. Data are presented as the mean \pm standard deviation. ${ }^{*} \mathrm{P}<0.05$ vs. Sham, ${ }^{\sharp} \mathrm{P}<0.05$ vs. IR, ${ }^{\$} \mathrm{P}<0.05$ vs. IR + GRb1. 8-iso-PGF $2 \alpha, 8$-iso-prostaglandin $\mathrm{F}_{2 \alpha}$; GRb1, ginsenoside Rb1; IR, ischemia/reperfusion; MDA, malondialdehyde; Sham, sham-operated group; SOD, superoxide dismutase; WM, Wortmannin.

The mechanisms underlying IIR injury are complex. It has been demonstrated that the intestinal mucosal barrier serves an important role in intestinal function (41); acute IIR may disrupt normal intestinal structure and increase the permeability of the barrier. D-lactate is produced by various bacteria in the gastrointestinal (GI) tract; as mammals lack the required enzymes for D-lactate degradation, increased serum levels of D-lactate serve as biomarkers of damage to the intestinal mucosa (42). DAO is a highly active intracellular enzyme that primarily resides in the cytoplasm of cells of the intestinal villus, and the serum expression levels of DAO can also increase following impairments in intestinal mucosal barrier function (43). Numerous studies have indicated that combined quantification of the levels of D-lactate and DAO in peripheral blood may indicate structural and functional alterations in intestinal mucosa permeability with more reliability than individual markers in certain GI diseases $(44,45)$. Endotoxins are structural molecules in the walls of gram-negative bacilli and potently induce a variety of disorders, including sepsis, infectious shock and gut-derived bacteremia (46). Yuan et al (47) reported that intestinal mucosal barriers are damaged following liver transplantation in rats due to intestinal congestion; enterogenous endotoxins can enter the bloodstream and be transported to other organs, leading to multiorgan dysfunction. In the present study, the serum levels of D-lactate, DAO and endotoxin were determined in a rat model of IIR. The results suggested that permeabilization of the intestinal mucosal barrier may be an important factor underlying intestinal injury during IIR. Additionally, pre-treatment with GRb1 significantly decreased the serum levels of D-lactate, DAO and endotoxin following IIR, suggesting that it improved the integrity of the intestinal mucosal barrier.

Previous studies involving rodent models of IIR have reported that IIR injury leads to the damage of nearby and remote organs via the secretion of proinflammatory cytokines, and the induction of oxidative stress and apoptosis following mast cell degranulation $(48,49)$, thereby adversely affecting disease prognosis. IIR injury is characterized by a dysregulated inflammatory response, during which TNF- $\alpha$, IL-6 and IL-1 $\beta$ have been demonstrated to serve important roles, such as in leukocyte chemotaxis (50). In the present study, it was revealed that IIR decreased the intestinal activity of the antioxidative enzyme SOD, and increased the levels of oxidative stress mediated by the end products of lipid peroxidation, MDA and 8 -iso-PGF $2 \alpha$. This may represent a possible mechanism of IIR. Ginseng extract has been demonstrated to exhibit immunomodulatory properties in various diseases (51). Tan et al (34) reported that GRb1 induced potent anti-inflammatory effects in postoperative ileus and contributed to the recovery of GI motility. Oh et al (52) reported that GRb1 possessed antiphotoaging properties in skin by scavenging ROS, decreasing the expression levels of matrix metalloproteinase-2 and enhancing antioxidant activity in keratinocytes under ultraviolet B irradiation. In the present study, pre-treatment with GRb1 significantly increased the activity levels of SOD, and reduced the secretion of inflammatory cytokines and oxidative stress factors following IIR, including TNF- $\alpha$, IL- 6 , IL- $1 \beta$ and MDA; however, WM significantly attenuated the aforementioned anti-inflammatory and antioxidative effects of GRb1. Collectively, these findings were consistent with previous studies regarding the properties of GRb1, and indicated that cytoprotective mechanisms may underlie the beneficial effects of GRb1 during IIR injury.

The PI3K/Akt signaling pathway is crucial for the proliferation, differentiation, apoptosis and glucose transport of cells (53). Previous studies have reported that the PI3K/Akt signaling pathway serves an important role in IR injury in certain organs. Kai-lan and Si (29) reported that activation of the PI3K/Akt signaling pathway suppresses nuclear factor $\kappa$-light-chain-enhancer of activated B cells-mediated inflammation in a rodent model of IIR. Yin et al (54) demonstrated that hyperbaric oxygen preconditioning protects against myocardial IR injury via the induction of a PI3K/Akt/Nrf2-dependent antioxidant defensive system. These findings were consistent with the results of the present study; however, other studies reported pathological effects of PI3K activity during IR. Zhang et al (55) revealed that dexamethasone protects mice against renal IR injury by supressing PI3K/Akt-mediated inflammatory responses. Furthermore, Zhu et al (56) demonstrated that catalpol ameliorates renal IR injury in a rat model via downregulation of PI3K/Akt-endothelial nitric oxide synthase signaling and inflammation. Collectively, these findings reveal the complex roles of PI3K in health and disease. Oudit et al (57) reported that PI3K $\alpha$ and $\beta$ were physiological 
A

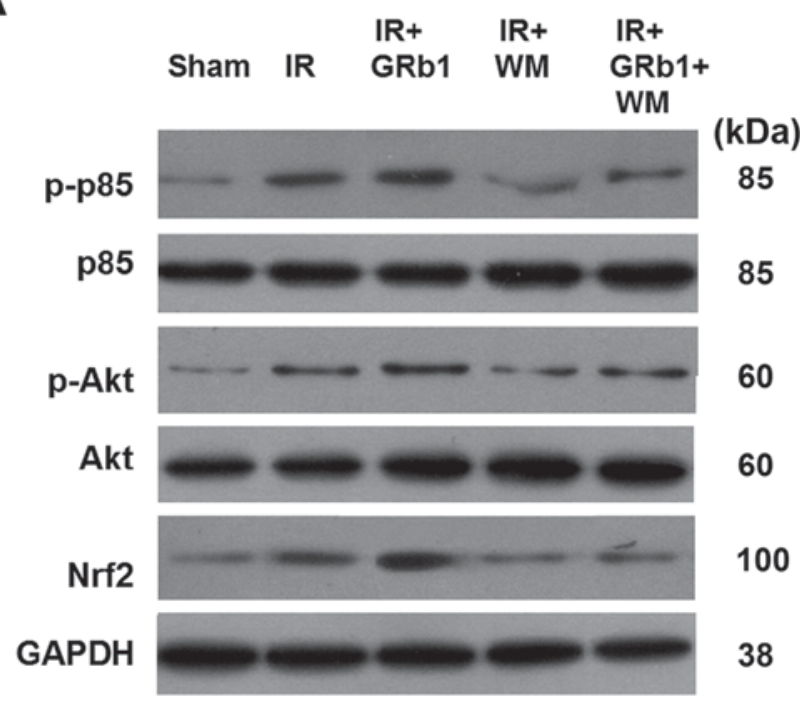

C

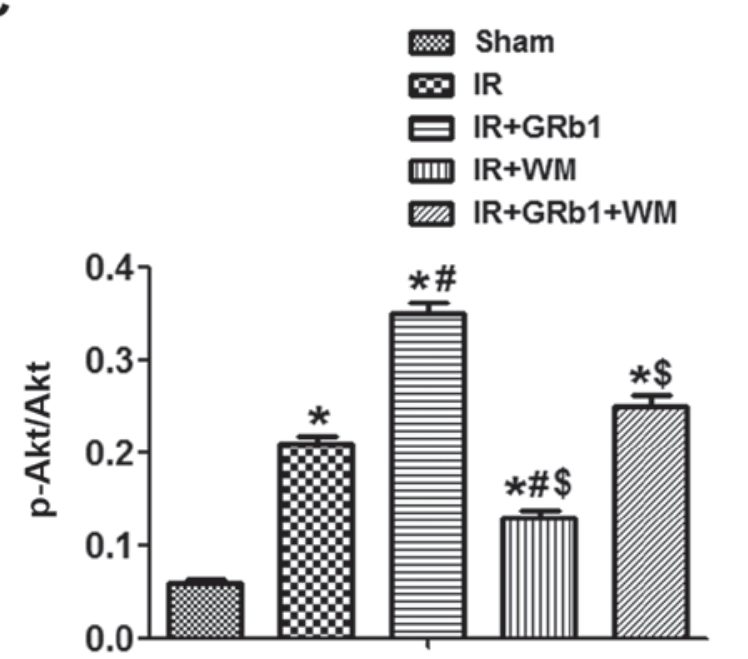

B

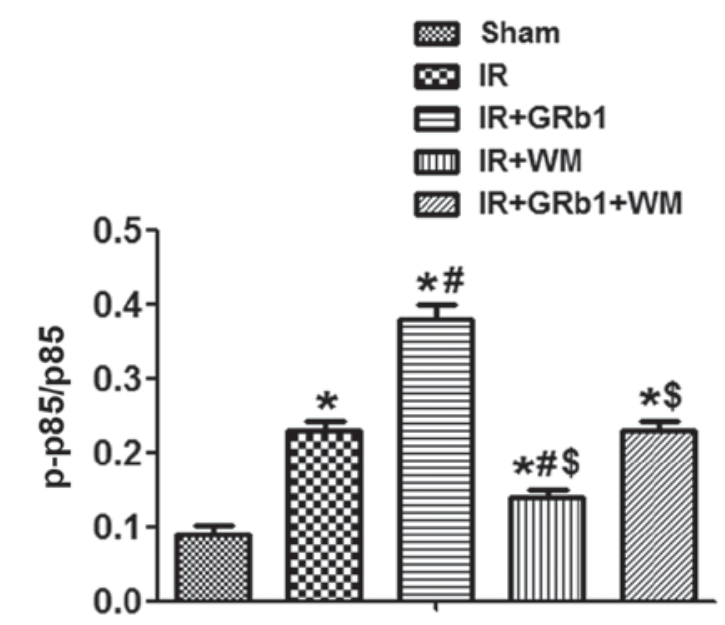

D
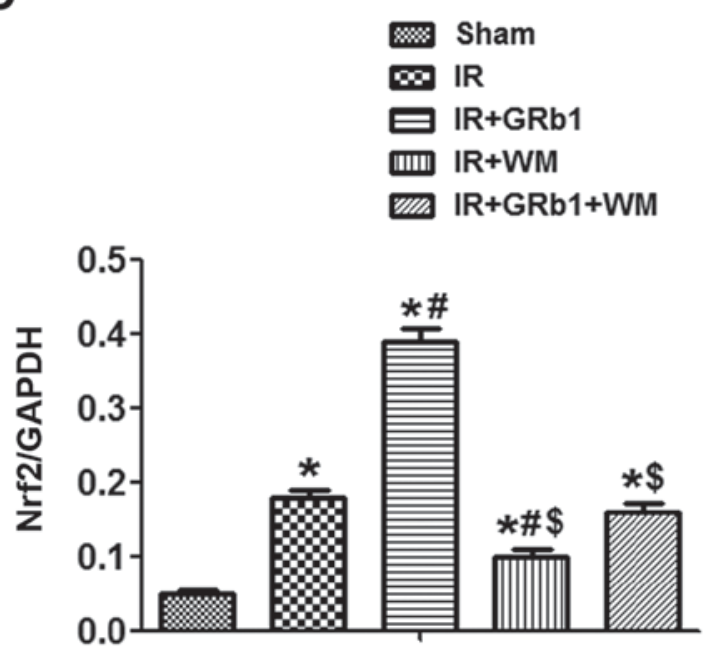

Figure 5. Pre-treatment with GRb1 activates the PI3K/Akt/Nrf2 signaling pathway during intestinal IR. (A) Intestinal expression levels of p-p85/p85, p-Akt/Akt, Nrf2 and GAPDH following Sham, or IR treatment in the presence or absence of $15 \mathrm{mg} / \mathrm{kg} \mathrm{GRb} 1$ and $0.6 \mathrm{mg} / \mathrm{kg} \mathrm{WM}$. Quantitation of the relative levels of (B) p-p85/p85, (C) p-Akt/Akt and (D) Nrf2 expression. N=6/group. Data are presented as the mean \pm standard deviation. " $\mathrm{P}<0.05$ vs. Sham, ${ }^{*} \mathrm{P}<0.05$ vs. IR, ${ }^{\$} \mathrm{P}<0.05$ vs. IR + GRb1. Akt, protein kinase B; GRb1, ginsenoside Rb1; IR, ischemia/reperfusion; Nrf2, nuclear factor erythroid 2-related factor 2; p85, regulatory subunit of Akt; p, phosphorylated; Sham, sham-operated group; WM, Wortmannin.

stimuli for adaptive cardiac hypertrophy, whereas PI3K $\gamma$ was a pathological stimulus for maladaptive hypertrophy. Separate PI3K isoforms may serve distinct roles in health and disease, and the regulation of distinct PI3K isoforms may elicit opposing effects. Therefore, future experiments are required to further investigate the mechanisms by which GRb1 affects the function of PI3K isoforms.

The transcription factor Nrf2 is an important downstream target of the PI3K/Akt pathway (58). In the present study, pre-treatment with WM exacerbated intestinal damage following IIR and significantly downregulated the expression of Nrf2 protein, whereas GRb1 increased the levels of Nrf2 expression. These results indicated that the PI3K/Akt/Nrf2 signaling pathway is involved in the protective effects of GRb1 against IIR injury.
The present study has certain limitations. The postoperative survival of rats subjected to IIR was not determined in the presence or absence of GRb1 and WM. Additionally, a PI3K activator was not included in the study to further investigate the involvement of PI3K/Akt signaling in the effects of GRb1 during IIR injury. Therefore, the aims of future experiments are to continue investigations into the exact mechanisms underlying the protective effects of GRb1.

In conclusion, the results of the present study suggested that GRb1 alleviates intestinal injury following IIR by activating the PI3K/Akt/Nrf2 pathway. The findings indicated that GRb1 may be a potential treatment for the prevention of IIR injury. Further research is required to evaluate the clinical efficacy of GRb1 and determine whether treatment with ginseng is beneficial in conditions associated with IIR. 


\section{Acknowledgements}

Not applicable.

\section{Funding}

The present study was supported by the Natural Science Foundation of Guangdong Province, China (grant no. 2014 A030313076 and 2016A030313232) and the Natural Science Foundation of China (grant no. 81501693).

\section{Availability of data and materials}

All data generated or analyzed during the present study are included in this published article.

\section{Authors' contributions}

SC and XL performed the ELISA and collected data. CC and YW contributed to sample collection. DL, PM and PH designed the study. SC and DL drafted the manuscript. All authors read and approved the final manuscript.

\section{Ethics approval and consent to participate}

The experimental protocol and design were approved by the Institutional Animal Care and Use Committee of Sun Yat-sen University, and were conducting following the Chinese guidelines for humane treatment of animals (17).

\section{Patient consent for publication}

Not applicable.

\section{Competing interests}

The authors declare that they have no competing interests.

\section{References}

1. Khadaroo RG, Churchill TA, Tso V, Madsen KL, Lukowski C and Salim SY: Metabolomic profiling to characterize acute intestinal ischemia/reperfusion injury. PLoS One 12: e0179326, 2017

2. Huang P, Liu D, Gan X, Zhang R, Gao W, Xia Z and Hei Z: Mast cells activation contribute to small intestinal ischemia reperfusion induced acute lung injury in rats. Injury 43: 1250-1256, 2012

3. Zhou J, Huang WQ, Li C, Wu GY, Li YS, Wen SH, Lei WL and Liu KX: Intestinal ischemia/reperfusion enhances microglial activation and induces cerebral injury and memory dysfunction in rats. Crit Care Med 40: 2438-2448, 2012.

4. Grootjans J, Lenaerts K, Buurman WA, Dejong $\mathrm{CH}$ and Derikx JP: Life and death at the mucosal-luminal interface: New perspectives on human intestinal ischemia-reperfusion. World J Gastroenterol 22: 2760-2770, 2016.

5. Hwang YP and Jeong HG: Ginsenoside Rb1 protects against 6-hydroxydopamine-induced oxidative stress by increasing heme oxygenase-1 expression through an estrogen receptor-related $\mathrm{PI} 3 \mathrm{~K} / \mathrm{Akt} / \mathrm{Nrf2}$-dependent pathway in human dopaminergic cells. Toxicol Appl Pharmacol 242: 18-28, 2010

6. Peng Y, Li SN, Pei X and Hao K: The multivariate regression statistics strategy to investigate content-effect correlation of multiple components in traditional Chinese medicine based on a partial least squares method. Molecules 23: E545, 2018.

7. Zheng Q, Bao XY, Zhu PC, Tong Q, Zheng GQ and Wang Y: Ginsenoside Rb1 for myocardial ischemia/reperfusion injury: Preclinical evidence and possible mechanisms. Oxid Med Cell Longev 2017: 6313625, 2017.
8. Liu D, Zhang H, Gu W, Liu Y and Zhang M: Neuroprotective effects of ginsenoside Rb1 on high glucose-induced neurotoxicity in primary cultured rat hippocampal neurons. PLoS One 8: e79399, 2013

9. Xue X, Wang L, Meng X, Jiao J and Dang N: Regulator of G protein signaling 4 inhibits human melanoma cells proliferation and invasion through the PI3K/AKT signaling pathway. Oncotarget 8: 78530-78544, 2017.

10. Huang L, Cao J, Cao L, Gao L, Yang Y and Xu L: Puerarin induces cell apoptosis in human chondrosarcoma cell line SW1353 via inhibition of the PI3K/Akt signaling pathway. Oncol Lett 14: 5585-5590, 2017.

11. Mellor P, Furber LA, Nyarko JN and Anderson DH: Multiple roles for the $\mathrm{p} 85 \alpha$ isoform in the regulation and function of PI3K signalling and receptor trafficking. Biochem J 441: 23-37, 2012.

12. Ren M, Wang $X, D u$ G, Tian J and Liu Y: Calycosin-7-O- $\beta$-D-glucoside attenuates ischemia-reperfusion injury in vivo via activation of the PI3K/Akt pathway. Mol Med Rep 13: 633-640, 2016.

13. Wang L, Yao Y, He R, Meng Y, Li N, Zhang D, Xu J, Chen O, Cui J, Bian J, et al: Methane ameliorates spinal cord ischemia-reperfusion injury in rats: Antioxidant, anti-inflammatory and anti-apoptotic activity mediated by Nrf2 activation. Free Radic Biol Med 103: 69-86, 2017.

14. Lee YJ, Jeong HY, Kim YB, Lee YJ, Won SY, Shim JH, Cho MK, Nam HS and Lee SH: Reactive oxygen species and PI3K/Akt signaling play key roles in the induction of Nrf2-driven heme oxygenase-1 expression in sulforaphane-treated human mesothelioma MSTO-211H cells. Food Chem Toxicol 50: 116-123, 2012.

15. Zhang R, Chae S, Lee JH and Hyun JW: The cytoprotective effect of butin against oxidative stress is mediated by the up-regulation of manganese superoxide dismutase expression through a PI3K/Akt/Nrf2-dependent pathway. J Cell Biochem 113: 1987-1997, 2012

16. Ministry of Science and Technology of China: Guideline on the humane treatment of laboratory animals. 2006

17. Gan X, Liu D, Huang P, Gao W, Chen X and Hei Z: Mast-cell-releasing tryptase triggers acute lung injury induced by small intestinal ischemia-reperfusion by activating PAR-2 in rats. Inflammation 35: 1144-1153, 2012.

18. Zhuang CL, Mao XY, Liu S, Chen WZ, Huang DD, Zhang CJ, Chen BC, Shen X and Yu Z: Ginsenoside Rb1 improves postoperative fatigue syndrome by reducing skeletal muscle oxidative stress through activation of the PI3K/Akt/Nrf2 pathway in aged rats. Eur J Pharmacol 740: 480-487, 2014.

19. Wang Z, YeZ, Huang G, Wang N, Wang E and Guo Q: Sevoflurane post-conditioning enhanced hippocampal neuron resistance to global cerebral ischemia induced by cardiac arrest in rats through PI3K/Akt survival pathway. Front Cell Neurosci 10: 271, 2016.

20. Petrat F, Swoboda S, de Groot H and Schmitz KJ: Quantification of ischemia-reperfusion injury to the small intestine using a macroscopic score. J Invest Surg 23: 208-217, 2010.

21. Jiang Y,Zhou Z, Meng QT, Sun Q, Su W, Lei S, Xia Z and Xia ZY: Ginsenoside Rbl treatment attenuates pulmonary inflammatory cytokine release and tissue injury following intestinal ischemia reperfusion injury in mice. Oxid Med Cell Longev 2015: 843721, 2015.

22. Cui YC, Pan CS, Yan L, Li L, Hu BH, Chang X, Liu YY, Fan JY, Sun K, -Li Q and Han JY: Ginsenoside Rb1 protects against ischemia/reperfusion-induced myocardial injury via energy metabolism regulation mediated by RhoA signaling pathway. Sci Rep 7: 44579, 2017.

23. Xun W, Shi L, Zhou H, Hou G, Cao T and Zhao C: Effects of curcumin on growth performance, jejunal mucosal membrane integrity, morphology and immune status in weaned piglets challenged with enterotoxigenic Escherichia coli. Int Immunopharmacol 27: 46-52, 2015.

24. Tornai T, Palyu E, Vitalis Z, Tornai I, Tornai D, Antal-Szalmas P, Norman GL, Shums Z, Veres G, Dezsofi A, et al: Gut barrier failure biomarkers are associated with poor disease outcome in patients with primary sclerosing cholangitis. World J Gastroenterol 23: 5412-5421, 2017.

25. Lin ZL, Tan SJ, Cheng MH, Zhao CY, Yu WK, He YL, Li J and Li N: Lipid-rich enteral nutrition controls intestinal inflammation, improves intestinal motility and mucosal barrier damage in a rat model of intestinal ischemia/reperfusion injury. J Surg Res 213: 75-83, 2017.

26. Ge M, Gan X, Liu D, Zhang W, Gao W, Huang P and Hei Z: Time-course analysis of counts and degranulation of mast cells during early intestinal ischemia-reperfusion injury in mice. Mol Med Rep 8: 401-406, 2013. 
27. Zhao W, Zhou S, Yao W, Gan X, Su G, Yuan D and Hei Z: Propofol prevents lung injury after intestinal ischemia-reperfusion by inhibiting the interaction between mast cell activation and oxidative stress. Life Sci 108: 80-87, 2014.

28. Takayama G, Ohtani M, Minowa A, Matsuda S and Koyasu S Class I PI3K-mediated Akt and ERK signals play a critical role in FceRI-induced degranulation in mast cells. Int Immunol 25 215-220, 2013.

29. Kai-lan W and Si Z: Pretreatment with erythropoietin attenuates intestinal ischemia reperfusion injury by further promoting PI3K/Akt signaling activation. Transplant Proc 47: 1639-1645, 2015.

30. Kim JH, Kim J, Chun J, Lee C, Im JP and Kim JS: Role of iRhom 2 in intestinal ischemia-reperfusion-mediated acute lung injury. Sci Rep 8: 3797, 2018.

31. Liu D, Gan X, Huang P, Chen X, Ge M and Hei Z: Inhibiting tryptase after ischemia limits small intestinal ischemia-reperfusion injury through protease-activated receptor 2 in rats J Trauma Acute Care Surg 73: 1138-1144, 2012.

32. Mancuso C and Santangelo R: Panax ginseng and Panax quinquefolius: From pharmacology to toxicology. Food Chem Toxicol 107: 362-372, 2017.

33. Cheng $\mathrm{Z}$ and $\mathrm{Li} \mathrm{L}$ : Ginsenoside $\mathrm{Rg} 3$ ameliorates lipopolysaccharide-induced acute lung injury in mice through inactivating the nuclear factor- $\mathrm{\kappa} \mathrm{B}(\mathrm{NF}-\mathrm{\kappa B})$ signaling pathway. Int Immunopharmacol 34: 53-59, 2016.

34. Tan S, Yu W, Lin Z, Chen Q, Shi J, Dong Y, Duan K, Bai X, $\mathrm{Xu} \mathrm{L}, \mathrm{Li} \mathrm{J}$ and Li N: Anti-inflammatory effect of ginsenoside $\mathrm{Rb} 1$ contributes to the recovery of gastrointestinal motility in the rat model of postoperative ileus. Biol Pharm Bull 37: 1788-1794, 2014.

35. Li Y, Tang J, Khatibi NH, Zhu M, Chen D, Tu L, Chen L and Wang S: Treatment with ginsenoside rb1, a component of panax ginseng, provides neuroprotection in rats subjected to subarachnoid hemorrhage-induced brain injury. Acta Neurochir Suppl 110: 75-79, 2011.

36. Tan S, Zhou F, Li N, Dong Q, Zhang X, Ye X, Guo J, Chen B and Yu Z: Anti-fatigue effect of ginsenoside Rb1 on postoperative fatigue syndrome induced by major small intestinal resection in rat. Biol Pharm Bull 36: 1634-1639, 2013.

37. Li G, Qian W and Zhao C: Analyzing the anti-ischemia-reperfusion injury effects of ginsenoside Rb1 mediated through the inhibition of p38 $\alpha$ MAPK. Can J Physiol Pharmacol 94: 97-103, 2016.

38. Sun Q, Meng QT, Jiang Y and Xia ZY: Ginsenoside Rb1 attenuates intestinal ischemia reperfusion induced renal injury by activating Nrf2/ARE pathway. Molecules 17: 7195-7205, 2012.

39. Chen T, Xue H, Lin R and Huang Z: MiR-126 impairs the intestinal barrier function via inhibiting S1PR2 mediated activation of PI3K/AKT signaling pathway. Biochem Biophys Res Commun 494: 427-432, 2017.

40. Zhao LL, Hu GC, Zhu SS, Li JF and Liu GJ: Propofol pretreatment attenuates lipopolysaccharide-induced acute lung injury in rats by activating thephosphoinositide-3-kinase/Akt pathway. Braz J Med Biol Res 47: 1062-1067, 2014

41. Nier A, Engstler AJ, Maier IB and Bergheim I: Markers of intestinal permeability are already altered in early stages of non-alcoholic fatty liver disease: Studies in children. PLoS One 12: e0183282, 2017.

42. Li H, Chen Y, Huo F, Wang Y and Zhang D: Association between acute gastrointestinal injury and biomarkers of intestinal barrier function in critically ill patients. BMC Gastroenterol 17: 45, 2017.

43. Guo YY, Liu ML, He XD, Jiang CQ and Liu RL: Functional changes of intestinal mucosal barrier in surgically critical patients. World J Emerg Med 1: 205-208, 2010.

44. Jin X, Yu CH, Lv GC and Li YM: Increased intestinal permeability in pathogenesis and progress of nonalcoholic steatohepatitis in rats. World J Gastroenterol 13: 1732-1736, 2007.
45. Song WB, Lv YH, Zhang ZS, Li YN, Xiao LP, Yu XP, Wang YY, Ji HL and Ma L: Soluble intercellular adhesion molecule-1, D-lactate and diamine oxidase in patients with inflammatory bowel disease. World J Gastroenterol 15: 3916-3919, 2009.

46. Uramatsu M, Matsumoto T, Tateda K, Shibuya K, Miyazaki S, Horino T, Tanabe M, Sumiyama Y, Kusachi S and Yamaguchi K: Involvement of endotoxin in the mortality of mice with gut-derived sepsis due to methicillin-resistant Staphylococcus aureus. Microbiol Immunol 54: 330-337, 2010.

47. Yuan DD, Chi XJ, Jin Y, Li X, Ge M, Gao WL, Guan JQ, Zhang $\mathrm{AL}$ and Hei ZQ: Intestinal injury following liver transplantation was mediated by TLR/NF-kB activation-induced cell apoptosis. Mol Med Rep 13: 1525-1532, 2016.

48. Onal O, Yetisir F, Sarer AE, Zeybek ND, Onal CO, Yurekli B, Celik HT, Sirma A and Kilic M: Prophylactic ozone administration reduces intestinal mucosa injury induced by intestinal ischemia-reperfusion in the rat. Mediators Inflamm 2015: 792016, 2015

49. Zhao W, Gan X, Su G, Wanling G, Li S, Hei Z, Yang C and Wang H: The interaction between oxidative stress and mast cell activation plays a role in acute lung injuries induced by intestinal ischemia-reperfusion. J Surg Res 187: 542-552, 2014.

50. Scott JR, Cukiernik MA, Ott MC, Bihari A, Badhwar A, Gray DK, Harris KA, Parry NG and Potter RF: Low-dose inhaled carbon monoxide attenuates the remote intestinal inflammatory response elicited by hindlimb ischemia-reperfusion. Am J Physiol Gastrointest Liver Physiol 296: G9-G14, 2009.

51. Liu ZQ: Chemical insights into ginseng as a resource for natural antioxidants. Chem Rev 112: 3329-3355, 2012.

52. Oh SJ, Kim K and Lim CJ: Protective properties of ginsenoside Rb1 against UV-B radiation-induced oxidative stress in human dermal keratinocytes. Pharmazie 70: 381-387, 2015.

53. Huang CY, Hsiao JK, Lu YZ, Lee TC and Yu LC: Anti-apoptotic PI3K/Akt signaling by sodium/glucose transporter 1 reduces epithelial barrier damage and bacterial translocation in intestinal ischemia. Lab Invest 91: 294-309, 2011.

54. Yin X, Wang X, Fan Z, Peng C, Ren Z, Huang L, Liu Z and Zhao K: Hyperbaric oxygen preconditioning attenuates myocardium ischemia-reperfusion injury Through upregulation of heme oxygenase 1 expression: PI3K/Akt/Nrf2 pathway involved. J Cardiovasc Pharmacol Ther 20: 428-438, 2015.

55. Zhang J, Yao Y, Xiao F, Lan X, Yu C, Zhang Y, Jiang C, Yang J, Pei G,Li Y, et al: Administration of dexamethasone protects mice against ischemia/reperfusion induced renal injury by suppressing PI3K/AKT signaling. Int J Clin Exp Pathol 6: 2366-2375, 2013.

56. Zhu J, Chen X, Wang $\mathrm{H}$ and Yan Q: Catalpol protects mice against renal ischemia/reperfusion injury via suppressing PI3K/Akt-eNOS signaling and inflammation. Int J Clin Exp Med 8: 2038-2044, 2015.

57. Oudit GY, Sun H, Kerfant BG, Crackower MA, Penninger JM and Backx PH: The role of phosphoinositide- 3 kinase and PTEN in cardiovascular physiology and disease. J Mol Cell Cardiol 37: 449-471, 2004

58. Li W, Ma F, Zhang L, Huang Y, Li X, Zhang A, Hou C, Zhu Y and Zhu Y: S-Propargyl-cysteine exerts a novel protective effect on methionine and choline deficient diet-induced fatty liver via Akt/Nrf2/HO-1 pathway. Oxid Med Cell Longev 2016: 4690857, 2016.

This work is licensed under a Creative Commons Attribution-NonCommercial-NoDerivatives 4.0 International (CC BY-NC-ND 4.0) License. 